\section{Re: Myocardial Infarction Associated with Adderall XR and Alcohol Use in a Young Man}

To the Editor: I appreciated the information in this article, ${ }^{1}$ as one who specializes in the diagnosis and treatment of attention deficit/hyperactivity disorder. I am curious whether the drug screen urinalysis performed in this case distinguished between amphetamines and methamphetamine; the article noted the patient's sample was positive for methamphetamine.

One patient of mine experienced legal problems when a routine urinalysis in the workplace resulted in positives for both amphetamine and methamphetamine; she was taking plain dextroamphetamine for her attention deficit disorder. The head of the testing laboratory assured me that their test distinguished between these agents and that a positive methamphetamine result suggested abuse whereas the positive result for amphetamines in her case was expected.

I believe the distinction is important to make in order to identify what may be a major complicating condition in a patient, namely substance abuse involving methamphetamine. In the case cited, substance abuse is evident for alcohol, but not for methamphetamines necessarily (though the Adderall was being misused).

I am unaware whether amphetamines metabolize to methamphetamine in the body, resulting in a "false positive" for methamphetamine solely from the use of amphetamines in an appropriate manner. With some drug monitoring systems making the distinction between the two, it seems unlikely.

I welcome any comments on the above.

David Pomeroy, MD, FAAFP

Brain Health NW

Bellevue, Washington

drdave@brainhealthnw.com

\section{Reference}

1. Jiao X, Velez S, Ringstad J, Eyma V, Miller D, Bleiberg M. Myocardial infarction associated with Adderall XR and alcohol use in a young man. J Am Board Fam Med 2009; 22:197-201.

doi: 10.3122/jabfm.2009.05.090048

The above letter was referred to the author of the article in question, who offers the following reply.

\section{Response: Re: Myocardial Infarction Associated with Adderall XR and Alcohol Use in a Young Man}

To the Editor: Thank you for the comment. The drug screening test cited in the article is the standard 9-panel urine screening test. It doesn't distinguish between am- phetamine and methamphetamine and reports as methamphetamine. There is, however, a more specialized test that can detect only methamphetamine. To my knowledge, methamphetamine is not one of intermediate metabolites of either amphetamine or dextroamphetamine.

Xiangyang Jiao, MD

Monarch Health

Columbus, Ohio

xyjiao111@yahoo.com

doi: 10.3122/jabfm.2009.05.090068

\section{Re: Effectiveness of Vitamin $B_{12}$ in Treating Recurrent Aphthous Stomatitis: A Randomized, Double-Blind, Placebo- Controlled Trial}

To the Editor: I read with interest the original research article, "Effectiveness of Vitamin $\mathrm{B}_{12}$ in Treating Recurrent Aphthous Stomatitis: A Randomized, Double-Blind, Placebo-Controlled Trial" by Volkov et al. ${ }^{1}$ As noted, this study suggests that daily $1000 \mathrm{mcg}$ cyanocobalamin placed under the tongue may be preventive for recurrent aphthous stomatitis (RAS) after 5 months of use. Cyanocobalamin is not bioactive and must first be converted in the body to a bioactive form before it can be of benefit.

I would like to draw your attention to another study published by myself and Jeff Haley titled "Effect of Bioactive $B_{12}$ in Adhering Discs on Aphthous Ulcers." This study, published October 2008 in Inside Dentistry with commentary by Howard E. Strassler, DMD, ${ }^{2}$ suggests that $500 \mathrm{mcg}$ bioactive methylcobalamin, when delivered daily into saliva via adherent discs that are adhered to the buccal side of a tooth and allow for a time release over 20 to 40 minutes (Avamin Melts, OraHealth, Bellevue, WA), may also be effective with respect to perceived improvement (reduced pain and duration) but with this occurring in as little as 4 weeks. This pilot study included a double-blinded, placebo-controlled trial $(n=15)$ and a nonblinded A-B-A assessment $(n=16)$.

It seems from these 2 studies that daily use of methylcobalamin time-released into saliva to allow for mucosal absorption may be an effective strategy for reducing the number, duration, and pain of recurrent aphthous ulcers, regardless of the level of underlying serum vita$\min \mathrm{B}_{12}$ levels.

Jeff Burgess, DDS, MSD

Oral Care Research Associates LLC Seattle, Washington jeffreyaburgess@hotmail.com

\section{References}

1. Volkov I, Rudoy I, Freud T, et al. Effectiveness of vitamin $B_{12}$ in treating recurrent aphthous stomatitis: a randomized, 